\title{
TEXTURE-DRIVEN CORONARY ARTERY PLAQUE CHARACTERIZATION USING WAVELET PACKET SIGNATURES
}

\author{
Amin Katouzian $^{1}$, Babak Baseri ${ }^{3}$, Elisa E. Konofagou ${ }^{1,2}$, Andrew F. Laine ${ }^{1,2}$ \\ Departments of ${ }^{1}$ Biomedical Engineering, and ${ }^{2}$ Radiology of Columbia University, New York, USA \\ ${ }^{3}$ School of Biomedical Sciences, University of Medicine and Dentistry of New Jersey, Newark, USA \\ ak2432@columbia.edu
}

\begin{abstract}
High-frequency ultrasound transducers are being widely used to generate high resolution, real time, cross-sectional images of the coronary arteries. In this paper, we present a robust unsupervised texture-derived technique based on multi-channel wavelet frames to delineate atherosclerotic plaque compositions. The intravascular ultrasound (IVUS) signals were acquired from coronary arteries dissected from 32 diseased cadaver hearts employing $40 \mathrm{MHz}$ mechanically rotating, single-element transducers. The wavelet packet representations were classified using a Kmeans clustering algorithm to generate IVUS-histology color maps (IV-HCMs) and categorize tissues in lipidic, fibrotic and calcified. Finally, two independent observers evaluated the results contrasting the histology images corresponding to the IV-HCMs. Our results show that the proposed algorithm may have great potential as an alternative to existing spectrum-based classification techniques.
\end{abstract}

Index Terms - Plaque characterization, Intravascular Ultrasound, Texture Analysis.

\section{INTRODUCTION}

Atherosclerotic plaques constitute the major cause of fatal acute coronary syndromes (ACS) that takes more than 250,000 lives in United States annually. Intravascular ultrasound (IVUS) has been the most favored and clinically approved imaging modality to detect the vulnerable plaques $[1,2]$. It has been shown that the ultrasound radio-frequency (RF) signals provide quantitative information on tissue microstructures [3]. Hence, the spectral based techniques have been developed in order to better characterize plaque compositions.

Nair et al introduced a supervised algorithm using seven spectral parameters (intercept, slope, mid-band-fit (MBF), minimum and maximum powers and their corresponding frequencies), extracted from the calibrated tissue spectrum in combination with a classification tree (CT) to differentiate plaque compositions using $30 \mathrm{MHz}$, unfocused, mechanically rotating transducers [4]. Currently, this methodology known as IVUS-virtual histology (IVUS-VH), has been implemented in the Volcano (Rancho Cordova, CA) IVUS clinical scanners using $20 \mathrm{MHz}$ phased array transducers. Kawasaki et al [5] combined integrated backscatter (IB) with IVUS (IB-IVUS) to generate tissue color maps and delineate lipid, fibrous and calcified tissues. In a recent study by our group, we introduced the full-spectrum analysis [6] as an alternative to IVUS-VH and IV-IB. We deployed a $40 \mathrm{MHz}$ rotating single-element transducer and classified tissues using both linear Fisher and k-NN classifiers.

The tissue spectra are known to vary even in the homogeneous regions due to 1 ) small changes in the angle of incidence of the ultrasound beam or variations in the geometric configuration of scatterers, 2) genuine changes in physical characteristics within the particular tissue type, and 3) variation in the transducer properties such as center frequency and bandwidth that may confound the characterization of tissue type from tissue signatures. This makes the tissue characterization problem more challenging especially for closely related tissues like fibro-lipidic and lipidic.

In this paper, we will present an effective texturederived atherosclerotic tissue characterization algorithm using discrete wavelet packet frame (DWPF) and twodimensional (2D) envelope detection introduced by Laine et al [7]. The extracted textural features are perfectly suited for classification and capture characteristics of the plaque with the highest correlation to histology. This would resolve one of the main limitations of IVUS, which is discrimination between fibrous and fatty tissues [8].

\section{IN VITRO DATA COLLECTION}

We collected the hearts from two sources: autopsy and transplant surgery, within 24 hours postmortem. The arteries were dissected from the heart with approximate size of $20-60 \times 20 \times 20 \mathrm{~mm}^{3}(l \times w \times h)$. We tied off the major 


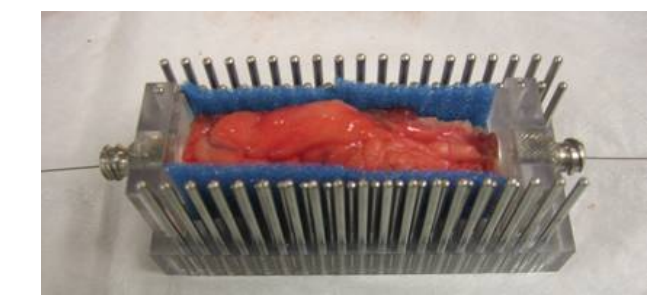

(a)

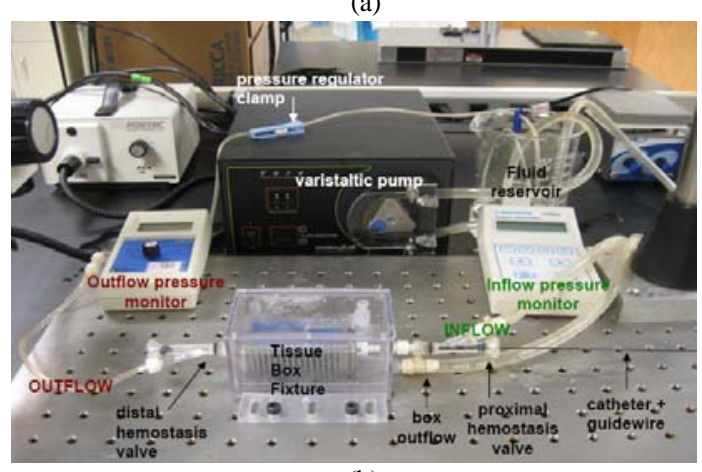

(b)

Figure1. Tissue cage fixture (a), experiment set up (b).

branches, attached the endplates to the distal and the proximal ends, and placed the segment into a tissue cage fixture, Figure 1(a). Circulating phosphate buffered saline (PBS) was used to ensure constant pressure $(100 \mathrm{mmHg})$ as well as flow and to maintain the artery physiologically opened at $37^{\circ}$ Celsius, Figure 1 (b). The $40 \mathrm{MHz}$ Boston Scientific (Fremont, CA) IVUS catheter was introduced and advanced on a 0.014 " guide wire and a complete automatic pullback was performed from the distal to the proximal side. The RF data was collected using two 12-bit Acqiris boards [Monroe, NY] and digitized at periodic time intervals, $T_{s}=2.5 n s\left(f_{s}=400 \mathrm{MHz}\right)$. The catheter pullback speed and the frame rate were set to $0.5 \mathrm{~mm} / \mathrm{s}$ and 30 frames $/ \mathrm{s}$, respectively. After imaging, the artery was pressure fixed with $10 \%$ buffered formaldehyde under $100 \mathrm{mmHg}$ for three hours followed by decalcification. The histology blocks were cut from distal to proximal every $2 \mathrm{~mm}$ (corresponding to 120 frames of the IVUS pullback) using the side rods. Blocks were then embedded in paraffin. For each block, three sections were used for histology. The first two sections were stained with hematoxylin and eosin (H\&E) and Movat Pentachrome, and the last section was kept unstained for future necessary staining (e.g., Sirius Red).

\section{METHODS}

Unlike discrete wavelet transform (DWT) and discrete wavelet packet transform (DWPT), the decompositions are translation invariant in DWPF. In other words, no decimation occurs between levels, Figure 2. Since the IVUS signals are sampled at the rate of $f_{s}$, it is more appropriate to consider the discrete signals that can be represented by a

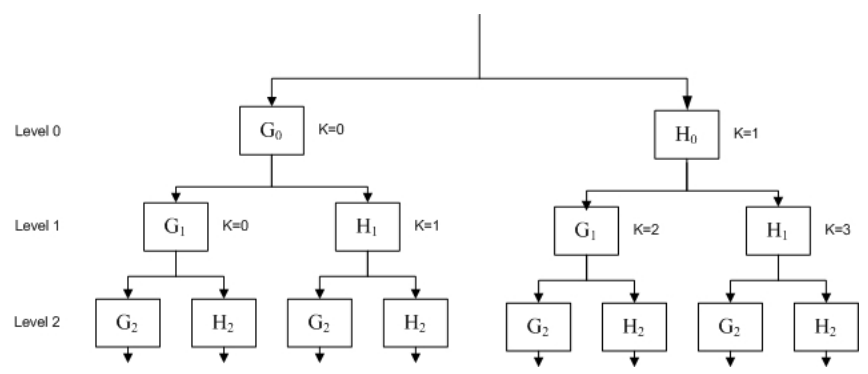

Figure 2. Tree structure for discrete wavelet packet frames DWPF) and associate indexes.

set of wavelet packet coefficients at the first scale $(l=0)$.

For the tree-structured scheme demonstrated in Figure 2, we can write:

$S_{2 k}^{l+1}(\omega)=G_{l}(\omega) S_{k}^{l}(\omega), S_{2 k+1}^{l+1}(\omega)=H_{l}(\omega) S_{k}^{l}(\omega)$,

where $S_{k}^{l}(\omega)$ is the Fourier transform of the frame coefficients at channel $k$ and level $l$.

\subsection{Filter Bank specification}

The multi-channel wavelet schematic in Figure 2 behaves like a filter bank with channel filters $\left\{F_{k}^{l}(\omega) \mid 0 \leq k \leq 2^{l}-1\right\}$, where $F_{k}^{l}(\omega)$ can be derived recursively as follows:

$F_{0}^{0}(\omega)=G_{0}(\omega), F_{1}^{0}(\omega)=H_{0}(\omega)$

$F_{2 k}^{l+1}(\omega)=G_{l+1}(\omega) F_{k}^{l}(\omega)=G_{0}\left(2^{l+1} \omega\right) F_{k}^{l}(\omega)$

$F_{2 k+1}^{l+1}(\omega)=H_{l+1}(\omega) F_{k}^{l}(\omega)=H_{0}\left(2^{l+1} \omega\right) F_{k}^{l}(\omega)$

Symmetry, frequency response, and boundary accuracy are the most crucial factors in selection of the highpass, $G_{l}(\omega)$, and the lowpass, $H_{l}(\omega)$, filters that directly impact the classification results. Hence, we selected Lemarie-Battle [9] wavelets that are symmetric (have linear phase response) and quadrature mirror filters (QMF). The wavelets using QMF cover exactly the frequency domain and satisfy the following property:

$\left|G_{0}(\omega)\right|^{2}+\left|H_{0}(\omega)\right|^{2}=\left|G_{0}(\omega)\right|+\left|H_{0}(\omega)\right|=1$

\subsection{Feature Extraction}

The features were extracted by processing of the IVUS signals for every frame in the $(r, \theta)$ domain. Each frame contains 256 lines that span 360 degree with 2048 samples per line. In order to have an optimized frame size in respect to the computational complexity and the textural resolution, we decimated and interpolated the signals in the axial and the lateral directions respectively to generate the square $M=512$ pixels frame. We chose a tensor product extension for each IVUS frame in which the channel filters were 


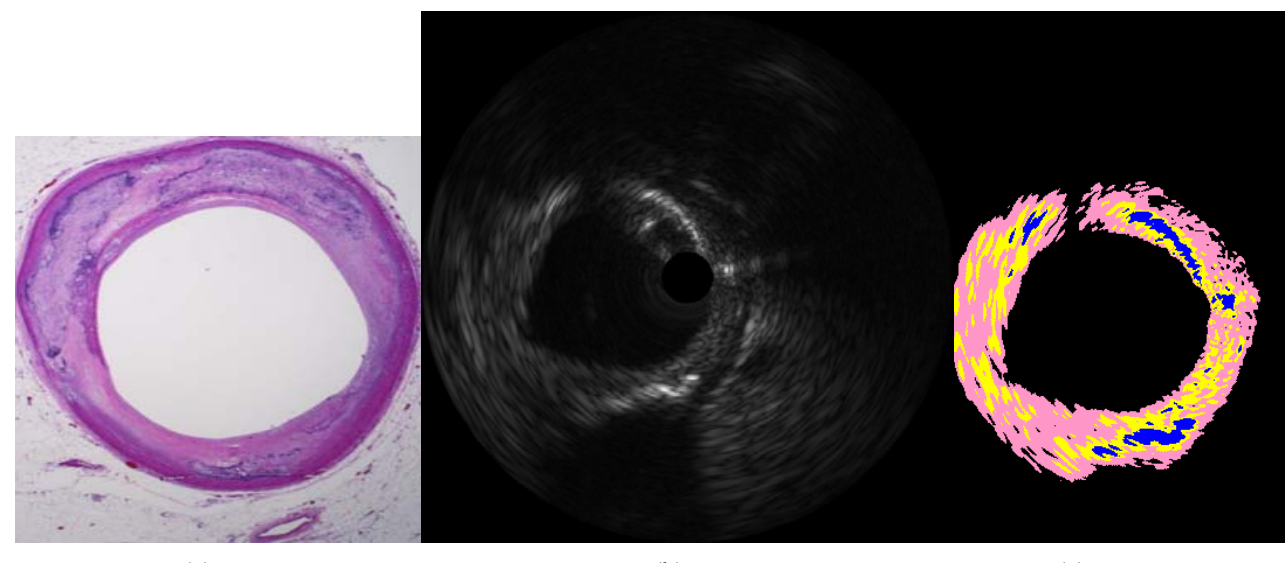

(a)

(b)

(c)

Figure 3. Movat Pentachrome histology image of cross section of interest (a) corresponding IVUS B-mode image (b), resulting IVUS-histology color map generated by the algorithm resulting IVUS-histology color map generated by the algorithm using Lemarie-Battle filters of order 18, decomposition level $\mathrm{L}=2$, number of classes $\mathrm{N}_{\mathrm{c}}=4$ (c). Blue, yellow and pink colors represent calcified, fibrotic and fibro-lipidic tissues.

denoted by $F_{i \times j}^{l}\left(\omega_{r}, \omega_{\theta}\right)=F_{i}^{l}\left(\omega_{r}\right) F_{j}^{l}\left(\omega_{\theta}\right)$. Such an extension would lead to the orientation selectivity in the decomposition tree.

We computed the envelope of output signals from channel filters and finally, the features matrix was constructed as follows:

$$
\vec{V}_{i, j}=\left\{e_{k, i, j}^{l} \mid 0 \leq k \leq\left(2^{l}-1\right), i, j=1, \ldots, M\right\}
$$

where $e_{k, i, j}^{l}$ represents the envelope value of pixel $(i, j)$ for the $k$-th component at level $l$.

\subsection{Classification}

The overall justification of in vivo real-time plaque characterization is performed by the interventional cardiologists through the use of classified tissues. For this reason, we chose the k-means clustering algorithm in order to classify the tissues and generate the IVUS-histology color maps (IV-HCMs). We utilized the unsupervised classifier to quantify the reliability of the extracted signatures. Our hypothesis is that if the classification results (IV-HCMs) driven by the unsupervised signatures preserve their high correlation with ground truth histology images, then the features can be used reliably in the training data set for supervised classification. We have categorized plaque components into four $N_{c}=4$ classes i.e., lipidic, fibrotic, calcified and background (no tissue).

For every representation matrix, $X_{M \times M}$, a label was assigned to each pixel by modulo $N_{c}$. We computed the center of clusters $\left\{\vec{C}_{\kappa} \mid 0 \leq \kappa \leq N_{c}-1\right\}$ by calculating the mean vector for each class. The pixel $\left\{x_{i, j} \mid i, j=1, \ldots, M\right\}$ was assigned to the class $\kappa$, if the Euclidean distance between the corresponding pixel and the class center $\vec{C}_{\kappa}$ was the closest. The centers of the clusters were updated in an iterative fashion by recomputing the relative mean vectors. The procedure was terminated once no change in labeling occurred.

\section{EXPERIMENTAL RESULTS}

Sixty four cross section of interest (CSI) collected from thirty two cadaver hearts, including 26 left anterior descending (LAD), 28 right coronary artery (RCA) and 20 left circumflex (LCX) segments that had more than $30 \%$ stenosis were examined. As we mentioned in the preceding section, we decimated the signals and used spline interpolation to generate 512-by-512 scan converted (Cartesian domain) B-mode images. For each frame, an expert manually segmented the plaque by tracing the vessel wall and lumen borders. The corresponding plaque signals were read and saved in a matrix with the same size of the IVUS image in the $(r, \theta)$ domain. We performed our algorithm on 512-by-512 matrices and selected LemarieBattle filters of order 18, decomposition level $L=2$ and number of classes $N_{c}=4$. Finally, the resulting classified images were mapped onto Cartesian plane. Figure 3 demonstrates a CSI, corresponding Movat Pentachrome histology image, and the IV-HCM. The blue, yellow and pink colors exhibit calcified, fibrotic, and lipidic plaque components, respectively.

\subsection{Quantification Process and Validation}

Although the histology image is the gold standard, its interpretation is subjective. For instance, one can simply categorize tissues as fibrotic, lipid pool, necrotic core and calcium, or one may differentiate between the levels of presence of fatty materials and add fibrolipid to compromise between fibrotic and lipid. Consequently, experts may evaluate the classification results differently. For this reason, we decided to use the scoring approach and asked 


\begin{tabular}{cccc} 
Tissue type & Histologist A & Histologist B & Mean \\
\hline \hline Fibrotic & 82.96 & 80.46 & 81.71 \\
\hline Lipidic & 83.70 & 81.83 & 82.76 \\
\hline Calcified & 82.60 & 88.43 & 85.51 \\
\hline Table 1. Percentage of correct classification evaluated by two histologists
\end{tabular}

Table 1. Percentage of correct classification evaluated by two histologists

two independent histologists to score each IV-HCM by looking at the corresponding histology slide. The accuracy of the characterization has been listed in Table 1 . The overall classification performance has been evaluated to be $81.71 \%, 82.76 \%$ and $85.51 \%$ for fibrotic, lipidic and calcified regions. The accuracy of IVUS-VH technique using autoregressive (AR) analysis in combination with a classification tree for $30 \mathrm{MHz}$ single element transducers has been reported to be $90.4 \%, 92.8 \%, 90.9 \%$ and $89.5 \%$ for the training data set and $79.7 \%, 81.2 \%, 92.8 \%$ and $85.5 \%$ for the test dataset in fibrotic, fibro-lipidic, calcified and calcified necrotic regions, respectively. In general, our results show that the algorithm performs fairly well especially in delineating between fibrotic and lipidic tissues. This would resolve one of the main limitations of IVUS, which is discrimination between fibrous and fatty tissues [10].

\section{SUMMARY AND CONCLUSION}

In this paper, we presented a reliable two-dimensional (2D) texture-derived atherosclerotic tissue characterization algorithm as an alternative to spectrum-based approaches like IV-VH, IB-IVUS and full-spectrum analysis. We processed the IVUS signals for each frame and extracted the textural features by using multi-channel wavelet packet analysis. The wavelet packet signatures were classified in an unsupervised fashion using K-means clustering algorithm.

The main advantage of the proposed algorithm is that it can reliably classify tissues regardless of the transducer center frequency or spectrum while inconsistency among the spectrum-derived features within the transducer's bandwidth still remains a major challenge. However, we were not able to detect the necrotic core tissues directly. Although both necrotic core tissues and lipid-rich pools have been recognized as markers for detection of vulnerable plaques, it is an advantage to have a classification algorithm that is able to detect the necrotic core in addition to fibrotic, lipidic and calcified tissues. In the future, we will attempt to extend the algorithm to supervised classification on raw IVUS backscattered signals and investigate this issue.

We will focus on two important aspects in future studies. First, the effects of blood on generated IV-HCMs and classification performance will be considered. In fact, it is an inevitable step to validate the reliability of the in vitro trained classifier for in vivo atherosclerotic plaque characterization. Secondly, the feasibility of tissue classification within the regions of acoustic shadowing behind calcification will be tested. Previously, we had reported that the tissue characterization in these regions remains difficult [11]. Our hypothesis was that sufficient spectral features would not exist due to signal attenuation in the densely calcified regions. This can be investigated through our proposed technique, since the background (no tissue) had been considered as one of the tissue types (classes).

\section{ACKNOWLEDGEMENTS}

The authors would like to thank Boston Scientific Inc. (Fremont, CA) for providing the transducers and acquisition systems. We also thank Jennifer Lisauskas and Stephen Sum, at InfraRedx (Burlington, MA), who developed the artery fixation cage and their invaluable help in collecting IVUS data. The histology samples were processed at CVPath (Gaithersburg, MD) under the supervision of Dr. R. Virmani.

\section{REFERENCES}

[1] P. Libby, "Molecular bases of the acute coronary syndromes," Circulation, vol. 91, pp2844-2850, 1995.

[2] R. Virmani, A. P. Burke, F. D. Kolodgie, A. Farb, "Pathology of the thin-cap fibroatheroma: A type of vulnerable plaque," J. Inteven. Cardiol., vol. 16, No. 3, pp. 267-272, 2003.

[3] F. L. Lizzi, M. Ostromogilsky, E. J. Feleppa, M. C. Rorke, M. M. Yaremko, "Relationship of Ultrasonic Spectral Parameters to Features of Tissue Microstructure," IEEE Trans. on UFFC, vol. 34, no. 3, pp. 319-329, 1986.

[4] A. Nair, B. D. Kuban, N. Obuchowski, D. G. Vince, “Assessing spectral algorithms to predict atherosclerotic plaque composition with normalized and raw intravascular ultrasound data. Ultrasound in Med. \& Biol., vol. 27, no. 10, pp. 1319-1331, 2001.

[5] M. Kawasaki, H. Takatsu, T. Noda, K. Sano, Y. Ito, K. Hayakawa, K. Tsuchiya, M. Arai, K. Nishigaki, G. Takemura,S. Minatoguchi,T. Fujiwara, H. Fujiwara," In Vivo Quantitative Tissue Characterization of Human Coronary Arterial Plaques by Use of Integrated Backscatter Intravascular Ultrasound and Comparison With Angioscopic Findings," Circulation, pp. 2487- 2492, May 2002.

[6] A. Katouzian, S. Sathyanarayana, B. Baseri, E. E. Konofagou, S. G. Carlier, "Challenges in Atherosclerotic Plaque Characterization with Intravascular Ultrasound (IVUS): From Data Collection to Classification," IEEE Trans. on Information Technology in Biomedicine, Special issue on IVUS (in press).

[7] A. F. Laine, J. Fan, "Frame representation for texture segmentation," IEEE Trans. Image Proc., vol. 5, no. 5, pp. 771-780.

[8] T. hiro, C. Y. Leung, S. De Guzman, V. J. Caiozzo, A. R. Fervid, H. Karimi, R. H. Helfant, J. M. Tobis, "Are soft echoes really soft? Intravascular ultrasound assessment of mechanical properties in human atherosclerotic tissue," American Heart J. vol. 133, pp. 1-7, 1997.

[9] S. G. Mallat, "A theory of multiresolution signal decomposition: The wavelet representation,” IEEE Trans. Patt. Anal. Mach. Intell., vol. 11, no. 7, pp. 674-693, 1989.

[10] T. hiro, C. Y. Leung, S. De Guzman, V. J. Caiozzo, A. R. Fervid, H. Karimi, R. H. Helfant, J. M. Tobis, "Are soft echoes really soft? Intravascular ultrasound assessment of mechanical properties in human atherosclerotic tissue," American Heart J. vol. 133, pp. 1-7, 1997.

[11] K. Tanaka, S. G. Carlier, A. Katouzian, G. Mintz, "Characterization of the intravascular ultrasound radiofrequency signal within regions of acoustic shadowing behind calcium,” J. Am. Coll. Cardiol., vol. 49, no. 9, Suppl. B, 29B, 2007. 